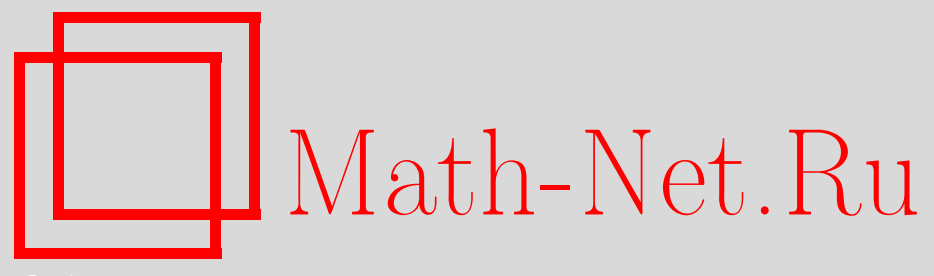

П. А. Бородин, Оценки расстояний до прямых и лучей от полюсов наипростейших дробей, ограниченных по норме $L_{p}$ на этих множествах, Матем. заметки, 2007, том 82, выпуск 6, 803-810

DOI: https://doi.org/10.4213/mzm4180

Использование Общероссийского математического портала Math-Net.Ru подразумевает, что вы прочитали и согласны с пользовательским соглашением http: //www. mathnet.ru/rus/agreement

Параметры загрузки:

IP : 3.85 .183 .62

26 апреля 2023 г., 11:34:10

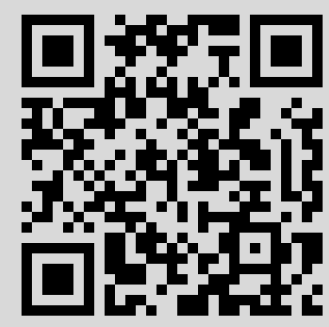


Том 82 выпуск 6 декабрь 2007

УДК 517.53

\section{Оценки расстояний до прямых и лучей от полюсов наипростейших дробей, ограниченных по норме $L_{p}$ на этих множествах}

\section{П. А. Бородин}

При каждом $p>1$ получена оценка снизу для расстояний до действительной оси от полюсов наипростейших дробей (т.е. логарифмических производных многочленов), ограниченных единицей в норме $L_{p}$ на этой оси, улучшающая первую оценку такого рода, полученную Данченко в 1994 г. При $p=2$ оценка оказывается точной. Аналогичные оценки получены для расстояний от полюсов наипростейших дробей до вершин углов и лучей.

Библиография: 8 названий.

1. Введение. Наипростейшей дробъю степени $n$ называется рациональная функция вида

$$
r_{n}(z)=\sum_{k=1}^{n} \frac{1}{z-a_{k}}=\frac{P^{\prime}(z)}{P(z)},
$$

где $\left\{a_{k}\right\}$ - точки комплексной плоскости $\mathbb{C}$, а $P(z)=\left(z-a_{1}\right) \cdots\left(z-a_{n}\right)$ - любой многочлен с нулями в этих точках.

В 1960-е г. Горин поставил следующую задачу. Пусть наипростейшая дробь $r_{n}(z)$ по модулю не превосходит 1 в каждой точке действительной оси $\mathbb{R}$. Как близко могут подходить к оси $\mathbb{R}$ полюсы $a_{k}$ этой дроби? Другими словами, стремятся ли к нулю величины

$$
d(n)=\inf \left\{\min \left|\operatorname{Im} a_{k}\right|:\left\|\sum_{k=1}^{n} \frac{1}{z-a_{k}}\right\|_{L_{\infty}(\mathbb{R})} \leqslant 1\right\}
$$

и, если стремятся, то с какой скоростью?

В работе [1] Данченко полностью решил эту задачу. Он показал, что

$$
d(n) \asymp \frac{\ln \ln n}{\ln n}, \quad n \rightarrow \infty
$$

(знак こ слабой эквивалентности означает, что отношение левой и правой частей ограничено сверху и снизу положительными постоянными). В [1] можно найти подробную библиографию и историю решения задачи Горина.

Работа выполнена при поддержке Российского фонда фундаментальных исследований (грант № 05-01-00962).

(C) П. А. Бородин, 2007 
Кроме того, Данченко в [1] исследовал аналогичную задачу для наипростейших дробей, ограниченных единицей по норме $L_{p}(\mathbb{R}), 1<p<\infty$. Приведем точную формулировку полученного им результата.

Пусть $1<p<\infty, 1 / p+1 / q=1, H_{p}\left(\mathbb{C}^{+}\right)$- пространство Харди в верхней полуплоскости $\mathbb{C}^{+}$, т.е. пространство голоморфных в $\mathbb{C}^{+}$функций $f$ с конечной нормой

$$
\|f\|_{H_{p}\left(\mathbb{C}^{+}\right)}=\sup _{a>0}\left(\int_{\mathbb{R}}|f(x+i a)|^{p} d x\right)^{1 / p} .
$$

Каждая функция $f \in H_{p}\left(\mathbb{C}^{+}\right)$имеет конечные угловые пределы $f(x)$ для почти всех $x \in \mathbb{R}$, образующие функцию $f(x) \in L_{p}(\mathbb{R})$. Пространство $H_{p}\left(\mathbb{C}^{+}\right)$содержит все наипростейшие дроби с полюсами в нижней полуплоскости.

Положим

$$
d(n, p)=\inf \left\{\min _{k} \operatorname{Im} a_{k}:\left\|\sum_{k=1}^{n} \frac{1}{z-a_{k}}+f(z)\right\|_{L_{p}(\mathbb{R})} \leqslant 1, \operatorname{Im} a_{k}>0, f \in H_{p}\left(\mathbb{C}^{+}\right)\right\} .
$$

Теорема А (В.И. Данченко). Для любъх $p \in(1, \infty)$ u $n \in \mathbb{N}$ имеет место неравенство

$$
d(n, p) \geqslant \frac{2^{q}(\sin (\pi / p))^{q}}{B(q-1 / 2,1 / 2)} \geqslant \frac{1}{p}\left(\sin \frac{\pi}{p}\right)^{q} 2^{q-1},
$$

где $B(\alpha, \beta)=\int_{0}^{1} t^{\alpha-1}(1-t)^{\beta-1} d t-$ бета-функция Эйлера (первая оценка, более точная, содержится в доказательстве теоремы $2 a$ работы [1], а вторая оценка, менее точная, указана в формулировке этой теоремы).

Примечательно, что правая часть здесь не зависит от $n$, так что полюсы наипростейших дробей $r_{n}(z)$ независимо от их количества не могут сколь угодно близко подходить к действительной оси, при условии $\left\|r_{n}\right\|_{L_{p}(\mathbb{R})} \leqslant 1$. Другими словами, слагаемые $1 /\left(z-a_{k}\right)$ наипростейшей дроби в случае $1<p<\infty$ (в отличие от случая $p=\infty$ ) не "интерферируют" и не могут в сумме дать маленькую $L_{p}$-норму на действительной оси, не удалившись от нее достаточно далеко.

Цель настоящей работы - уточнить оценку Данченко для величин $d(n, p)$ (теорема 1). Новая оценка лучше оценки Данченко при всех $p$ (теорема 2) и является точной при $p=2$ (следствие из теоремы 1). Кроме того, в работе получены (п. 4) оценки расстояний от полюсов наипростейших дробей $r_{n}(z)$ до вершин углов и лучей $\Lambda$ при условии $\left\|r_{n}\right\|_{L_{p}(\Lambda)} \leqslant 1$, а также показана связь этих оценок с теорией приближений наипростейшими дробями (п. 3).

\section{2. Оценка расстояний до прямой.}

Теорема 1. Для любых $p \in(1, \infty)$ u $n \in \mathbb{N}$ имеет место неравенство

$$
\frac{2^{2 q-2} \pi^{q-1}}{p^{q}} B\left(\frac{q}{2}, \frac{q}{2}\right) \leqslant d(n, p) \leqslant\left(\frac{\pi q 2^{2-p}}{p B(p / 2, p / 2)}\right)^{q-1}
$$

где $1 / p+1 / q=1, B(\alpha, \beta)=\int_{0}^{1} t^{\alpha-1}(1-t)^{\beta-1} d t-$ бета-функиия Эйлера.

ДокАЗАТЕЛЬСтво оценки снизу основывается на следующем простом факте, использовавшемся Косухиным (см. доказательство его теоремы 2 в работе [2]). Если 
$a$ и $b$ - два комплексных числа с ненулевой мнимой частью и $\operatorname{Im} a>0$, то

$$
\int_{\mathbb{R}} \frac{d x}{(x-a)(x-b)}= \begin{cases}\frac{2 \pi i}{a-b}, & \operatorname{Im} b<0 \\ 0, & \operatorname{Im} b>0\end{cases}
$$

Кроме того, для любой функции $f \in H_{p}\left(\mathbb{C}^{+}\right)$и любого числа $b \in \mathbb{C}, \operatorname{Im} b<0$, справедливо равенство

$$
\int_{\mathbb{R}} \frac{f(x)}{x-b} d x=0
$$

(см. упражнение 2 к главе 2 в книге [3]).

Возьмем произвольную функцию

$$
F(z)=\sum_{k=1}^{n} \frac{1}{z-a_{k}}+f(z)
$$

где $a_{k}=x_{k}+i \alpha_{k}$, все $\alpha_{k}>0, f \in H_{p}\left(\mathbb{C}^{+}\right)$, и пусть $\|F\|_{L_{p}(\mathbb{R})} \leqslant 1$. Без ограничения общности можно считать, что $a_{1}=\alpha i, \alpha>0$, и что $a_{1}$ - самая близкая к оси $\mathbb{R}$ точка из всех $a_{k}$, т.е. что $\alpha \leqslant \alpha_{k}, k=2, \ldots, n$.

Используя формулы (1) и (2), при любом $t>0$ получаем

$$
\int_{\mathbb{R}} \frac{F(x)}{x+t i} d x=\left|\frac{2 \pi i}{\alpha i+t i}+\sum_{k=2}^{n} \frac{2 \pi i}{a_{k}+t i}\right|=\left|\frac{2 \pi}{\alpha+t}+2 \pi \sum_{k=2}^{n} \frac{t+\alpha_{k}+i x_{k}}{\left(t+\alpha_{k}\right)^{2}+x_{k}^{2}}\right| \geqslant \frac{2 \pi}{\alpha+t} .
$$

С другой стороны, по неравенству Гёльдера

$$
\begin{aligned}
\left|\int_{\mathbb{R}} \frac{F(x)}{x+t i} d x\right| & \leqslant\|F\|_{L_{p}(\mathbb{R})} \cdot\left\|\frac{1}{x+t i}\right\|_{L_{q}(\mathbb{R})} \leqslant\left\|\frac{1}{x+t i}\right\|_{L_{q}(\mathbb{R})} \\
& =\left(\int_{\mathbb{R}} \frac{d x}{\left(x^{2}+t^{2}\right)^{q / 2}}\right)^{1 / q}=\frac{1}{t^{(q-1) / q}}\left(\int_{\mathbb{R}} \frac{d u}{\left(u^{2}+1\right)^{q / 2}}\right)^{1 / q}=: t^{-1 / p} c_{q} .
\end{aligned}
$$

Таким образом, при любом $t>0$ имеем

$$
\frac{2 \pi}{\alpha+t} \leqslant t^{-1 / p} c_{q} \quad \Rightarrow \quad \alpha \geqslant \frac{2 \pi}{c_{q}} t^{1 / p}-t=: g(t) .
$$

Функция $g(t)$ при $t>0$ достигает своего максимума в точке $t_{0}=\left(2 \pi /\left(p c_{q}\right)\right)^{q}$, откуда

$$
\begin{aligned}
\alpha & \geqslant g\left(t_{0}\right)=\frac{2 \pi}{c_{q}}\left(\frac{2 \pi}{p c_{q}}\right)^{q / p}-\left(\frac{2 \pi}{p c_{q}}\right)^{q}=\left(\frac{2 \pi}{p c_{q}}\right)^{q-1}\left(\frac{2 \pi}{c_{q}}-\frac{2 \pi}{p c_{q}}\right) \\
& =\left(\frac{2 \pi}{p c_{q}}\right)^{q-1} \cdot \frac{2 \pi}{q c_{q}}=\frac{2^{q} \pi^{q}}{p^{q-1} q c_{q}^{q}} .
\end{aligned}
$$

Согласно задаче 31.06 из [4]

$$
c_{q}^{q}=\int_{\mathbb{R}} \frac{d u}{\left(u^{2}+1\right)^{q / 2}}=B\left(\frac{1}{2}, \frac{q}{2}-\frac{1}{2}\right)=\frac{\Gamma(1 / 2) \Gamma((q-1) / 2)}{\Gamma(q / 2)}=\frac{\sqrt{\pi} \Gamma((q-1) / 2)}{\Gamma(q / 2)},
$$


где $\Gamma(x)=\int_{0}^{\infty} e^{-t} t^{x-1} d t-$ гамма-функция Эйлера. По формуле удвоения Лежандра (см. $[5 ;$ с. 867])

поэтому

$$
\Gamma\left(\frac{q-1}{2}\right) \Gamma\left(\frac{q}{2}\right)=\sqrt{\pi} 2^{2-q} \Gamma(q-1),
$$

$$
c_{q}^{q}=\frac{\pi 2^{2-q} \Gamma(q-1)}{\Gamma^{2}(q / 2)}=\frac{\pi 2^{2-q} \Gamma(q)}{(q-1) \Gamma^{2}(q / 2)}=\frac{p \pi 2^{2-q}}{q B(q / 2, q / 2)} .
$$

Подставляя это выражение в (3), получаем

$$
\alpha \geqslant \frac{2^{q} \pi^{q} q B(q / 2, q / 2)}{p^{q-1} q p \pi 2^{2-q}}=\frac{2^{2 q-2} \pi^{q-1}}{p^{q}} B\left(\frac{q}{2}, \frac{q}{2}\right),
$$

что и доказывает оценку снизу в утверждении теоремы 1.

Для доказательства оценки сверху найдем такое число $y_{0}>0$, что

$$
1=\left\|\frac{1}{x-i y_{0}}\right\|_{L_{p}(\mathbb{R})}^{p}=\int_{\mathbb{R}} \frac{d x}{\left(x^{2}+y_{0}^{2}\right)^{p / 2}}=y_{0}^{1-p} \int_{\mathbb{R}} \frac{d u}{\left(u^{2}+1\right)^{p / 2}}=y_{0}^{1-p} c_{p}^{p} .
$$

Имеем

$$
y_{0}=\left(c_{p}^{p}\right)^{1 /(p-1)}=\left(c_{p}^{p}\right)^{q-1}=\left(\frac{q \pi 2^{2-p}}{p B(p / 2, p / 2)}\right)^{q-1}
$$

(см. выше вычисление формулу (5)).

По определению $d(n, p) \leqslant y_{0}$, откуда и следует оценка сверху в утверждении теоремы 1 .

Теорема 1 доказана.

СлеДСтвиЕ. Для любого $n \in \mathbb{N}$ имеет место равенство

$$
d(n, 2)=\pi
$$

Отметим, что теорема А дает оценку $d(n, 2) \geqslant 1$.

Теорема 2. При каждом $p \in(1, \infty)$ оценка снизу в теореме 1 лучше, чем в теореме $\mathrm{A}$.

ДокАзАтЕЛьство. Надо показать, что функция

$$
h(p)=\left(\frac{2^{2 q-2} \pi^{q-1}}{p^{q}} B\left(\frac{q}{2}, \frac{q}{2}\right)\right):\left(\frac{1}{p}\left(\sin \frac{\pi}{p}\right)^{q} 2^{q-1}\right)=\frac{2^{q-1} \pi^{q-1} B(q / 2, q / 2)}{p^{q-1}(\sin (\pi / p))^{q}}
$$

больше 1 при каждом $p>1$. Рассмотрим несколько случаев.

Из формул (4) и (5) в доказательстве теоремы 1 получаем

$$
B\left(\frac{q-1}{2}, \frac{1}{2}\right)=\frac{p \pi 2^{2-q}}{q B(q / 2, q / 2)}
$$

откуда

$$
h(p)=\frac{\pi^{q}}{p^{q-1} q(\sin (\pi / p))^{q}} \geqslant \frac{\pi^{q}}{p^{q-1} q}=\frac{\pi^{q}}{(1+1 /(q-1))^{q-1} q}>\frac{\pi^{q}}{e q} \geqslant \frac{\pi^{1}}{e \cdot 1}>1
$$

(предпоследнее неравенство следует из того, что функция $f(q)=\pi^{q} / e q$ возрастает при $q \in[1, \infty))$.

Теорема 2 доказана. 
3. Связь с теорией приближений. При доказательстве теоремы 1 получалось, что любая наипростейшая дробь $r_{n}(z)$ с полюсами вне действительной оси $\mathbb{R}$ удовлетворяет неравенству

$$
\operatorname{Re} \int_{\mathbb{R}} \frac{r_{n}(x)}{x+i t} d x \geqslant 0
$$

при любом $t>0$. Это означает, что в пространстве $L_{p}(\mathbb{R}), 1<p<\infty$, наипростейшие дроби содержатся в полупространстве

$$
\Pi(t)=\left\{f \in L_{p}(\mathbb{R}): \operatorname{Re} \int_{\mathbb{R}} \frac{f(x)}{x+i t} d x \geqslant 0\right\},
$$

а значит, не плотны в $L_{p}(\mathbb{R})$ (этот факт, впрочем, следует из теоремы А и уже отмечался в [2]). В случае $p=2$ оценки сверху и снизу в теореме 1 смыкаются (см. следствие из теоремы 1 ), и из способа получения этих оценок дополнительно вытекает, что для любого $a \in \mathbb{C} \backslash \mathbb{R}$ функция $-1 /(x-a)$ не может быть приближена в $L_{2}(\mathbb{R})$ наипростейшими дробями лучше, чем нулем.

В то же время из результатов работ [6], [7] следует, что для любой ограниченной незамкнутой гладкой кривой $\gamma$ без самопересечений наипростейшие дроби плотны в пространстве $C(\gamma)$ непрерывных на $\gamma$ функций, а значит, и в любом пространстве $L_{p}(\gamma)$ с $p \in[1, \infty)$.

Возникает задача о плотности наипростейших дробей в пространствах $L_{p}(\gamma)$, $1<p<\infty$, для неограниченных кривых $\gamma$ без самопересечений. Рассмотрим простейший класс таких кривых - углы

$$
\Lambda(\alpha)=\left\{r e^{i \varphi}: r \geqslant 0, \varphi=0 \text { или } \varphi=\alpha\right\}, \quad \text { где } \quad \alpha \in[0, \pi] .
$$

В частности, в случае $\Lambda(\pi)=\mathbb{R}$, как уже отмечалось, наипростейшие дроби не плотны во всех пространствах $L_{p}(\mathbb{R}), 1<p<\infty$. Это утверждение остается справедливым и при всех $\alpha \in(0, \pi)$. Как показал Косухин (теорема 2 работы [2]), в пространстве $L_{p}(\Lambda(\alpha))$ при $\alpha \in(0, \pi)$ наипростейшие дроби содержатся в полупространстве

$$
\Pi(\alpha)=\left\{f \in L_{p}(\Lambda(\alpha)): \operatorname{Re} \int_{\Lambda(\alpha)} \frac{f(z)}{z^{\pi / \alpha}+i} d z \geqslant 0\right\},
$$

где $z^{\pi / \alpha}$ - такая аналитическая в области $\Omega(\alpha)=\left\{r e^{i \varphi}: r>0, \varphi \in(0, \alpha)\right\}$ и непрерывная вплоть до $\Lambda(\alpha)$ ветвь многозначной функции $e^{(\pi / \alpha) \log z}$, что $\operatorname{Im} z^{\pi / \alpha}>0$ для любого $z \in \Omega(\alpha)$. Поскольку функция $1 /\left(z^{\pi / \alpha}+i\right)$ принадлежит пространству $L_{q}(\Lambda(\alpha))$ при любом $q \geqslant 1, \Pi(\alpha)$ - собственное подпространство в $L_{p}(\Lambda(\alpha))$, а значит, наипростейшие дроби не плотны в $L_{p}(\Lambda(\alpha)), 1<p<\infty$, а также в пространстве функций $f$, непрерывных на $\Lambda(\alpha)$ и таких, что $f(z) \rightarrow 0$ при $z \rightarrow \infty$, с равномерной нормой.

Однако этот результат удается лишь частично распространить на случай $\Lambda(0)=$ $[0, \infty)$

Теорема 3. Наипростейшие дроби не плотны в пространстве $L_{p}[0, \infty)$ при любом $p \in(1,2)$.

ДокАЗАТЕЛЬСтво. Для любого числа $a \in \mathbb{C} \backslash[0, \infty)$ имеем

$$
\int_{0}^{\infty} \frac{\sqrt{x}}{x+1} \cdot \frac{1}{x-a} d x=\frac{\pi i}{\sqrt{a}+i}
$$


(см. задачу 28.20 из [4]), где непрерывная ветвь $\sqrt{z}$ в $\mathbb{C} \backslash[0, \infty)$ выбирается так, что $\operatorname{Im} \sqrt{z}>0$ для любого $z \in \mathbb{C} \backslash[0, \infty)$. Поскольку $\operatorname{Re} \pi i /(\sqrt{a}+i)>0$ для любого $a \in \mathbb{C} \backslash$ $[0, \infty)$, а функция $\sqrt{x} /(x+1)$ принадлежит пространству $L_{q}[0, \infty)$ при любом $q>2$, получаем, что наипростейшие дроби содержатся в собственном полупространстве во всех пространствах $L_{p}[0, \infty), 1<p<2$, а значит, не плотны в каждом из этих пространств.

Теорема 3 доказана.

По-видимому, не существует такой функции $g \in L_{2}[0, \infty)$, для которой неравенство $\operatorname{Re} \int_{0}^{\infty} g(x) /(x-a) \geqslant 0$ справедливо при любом $a \in \mathbb{C} \backslash[0, \infty)$. Возможно, верно и более сильное утверждение: наипростейшие дроби плотны в пространстве $L_{2}[0, \infty)$.

4. Оценки расстояний до углов и луча. Отмеченные в предыдущем пункте неравенства можно использовать для оценки расстояний от полюсов наипростейших дробей $r(z)$ до углов $\Lambda(\alpha)$ или луча $[0, \infty)$ при условии ограниченности $\|r\|$ в соответствующем пространстве $L_{p}$. Приведем несколько таких оценок в специальных случаях - когда рассматриваемый полюс берется на биссектрисе (внутренней или внешней) угла $\Lambda(\alpha)$ или, соответственно, на луче $(-\infty, 0)$, и при этом оценивается расстояние от этого полюса до нуля, т.е. до вершины угла $\Lambda(\alpha)$ или луча $[0, \infty)$.

Teоpema 4. Пусть $\alpha \in(0, \pi], p \in(1, \infty], a>0 u$

$$
r(z)=\frac{1}{z-a e^{i \alpha / 2}}+\sum_{k=1}^{n} \frac{1}{z-a_{k}}
$$

- наипростейшая дробъ с полюсами вне угла $\Lambda(\alpha)$, у которой $\|r\|_{L_{p}(\Lambda(\alpha))} \leqslant 1$. Тогда

$$
a \geqslant \frac{2^{q} \pi(\pi q-\alpha)^{q-\alpha / \pi}}{q^{q} \alpha^{1-\alpha / \pi} B(\alpha /(2 \pi),(q \pi-\alpha) /(2 \pi))} .
$$

ДокАЗАТЕЛЬСтво проводится аналогично доказательству оценки снизу в теореме 1. Из неравенств

$$
\left|\int_{\Lambda(\alpha)} \frac{r(z)}{z^{\pi / \alpha}+i t} d z\right| \geqslant\left|\int_{\Lambda(\alpha)} \frac{d z}{\left(z-a e^{i \alpha / 2}\right)\left(z^{\pi / \alpha}+i t\right)}\right|=\frac{2 \pi}{a^{\pi / \alpha}+t}
$$

(здесь используется отмеченное в п. 3 утверждение Косухина) и

$$
\begin{aligned}
\left|\int_{\Lambda(\alpha)} \frac{r(z)}{z^{\pi / \alpha}+i t} d z\right| & \leqslant\left\|\frac{1}{z^{\pi / \alpha}+i t}\right\|_{L_{q}(\Lambda(\alpha))} \\
& =t^{\alpha /(\pi q)-1}\left(\int_{\mathbb{R}} \frac{d u}{\left(|u|^{2 \pi / \alpha}+1\right)^{q / 2}}\right)^{1 / q}=: t^{\alpha /(\pi q)-1} d_{q}
\end{aligned}
$$

следует, что

$$
a^{\pi / \alpha} \geqslant \frac{2 \pi t^{1-\alpha /(\pi q)}}{d_{q}}-t
$$

Находя максимум правой части последнего неравенства при $t \geqslant 0$ и вычисляя

$$
d_{q}^{q}=\frac{\alpha}{\pi} B\left(\frac{\alpha}{2 \pi}, \frac{q}{2}-\frac{\alpha}{2 \pi}\right)
$$

(см. задачу 31.06 в [4]), получаем утверждение теоремы. 
Отметим, что при $\alpha=\pi$ оценка в теореме 4 совпадает с оценкой снизу в теореме 1 .

Teopema 5. Пусть $\alpha \in(0, \pi), a>0, \delta=\pi /(2 \pi-\alpha), 1<p<(2 \pi-\alpha) /(\pi-\alpha)$,

$$
r(z)=\frac{1}{z+a e^{i \alpha / 2}}+\sum_{k=1}^{n} \frac{1}{z-a_{k}}
$$

- наипростейшая дробъ с полюсами вне угла $\Lambda(\alpha)$, у которой $\|r\|_{L_{p}(\Lambda(\alpha))} \leqslant 1$. Тогда

$$
a \geqslant\left(\frac{2 \pi}{\delta q}\right)^{q} \frac{\delta(\delta q-1)^{q-1 / \delta}}{B(1 /(2 \delta),(q \delta-1) /(2 \delta))} .
$$

ДоКАЗАТЕЛЬСТво проводится аналогично доказательству предыдущей теоремы и использует неравенство.

$$
\operatorname{Re} \int_{\Lambda(\alpha)} \frac{1}{z^{\delta}-i t} \frac{1}{z-b} d z>0
$$

где $t>0, b \in \mathbb{C} \backslash \Lambda(\alpha)$, а $z^{\delta}$ - аналитическая в области $\Omega^{\prime}(\alpha)=\left\{r e^{i \varphi}: r>0, \varphi \in\right.$ $(\alpha, 2 \pi)\}$ и непрерывная вплоть до $\Lambda(\alpha)$ ветвь многозначной функции $e^{\delta \log z}$, переводящая $\Omega^{\prime}(\alpha)$ в нижнюю полуплоскость.

В случае $p \geqslant(2 \pi-\alpha) /(\pi-\alpha)$ полюсы наипростейших дробей $r(z)$, ограниченных единицей по норме $L_{p}(\Lambda(\alpha))$, по-видимому, могут сколь угодно близко подходить к вершине угла $\Lambda(\alpha)$ с внешней стороны.

Tеорема 6. Пусть $a>0,1<p<2$,

$$
r(z)=\frac{1}{z+a}+\sum_{k=1}^{n} \frac{1}{z-a_{k}}
$$

- наипростейшая дробъ с полюсами вне луча $[0, \infty)$, у которой $\|r\|_{L_{p}[0, \infty)} \leqslant 1$. Тогда

$$
a \geqslant \frac{4 \pi^{q}(q-2)^{q-1}}{q^{q+1} B(q / 2, q / 2)} .
$$

ДокАЗАТЕЛЬСтво аналогично доказательству теоремы 4 и использует неравенство

$$
\operatorname{Re} \int_{0}^{\infty} \frac{\sqrt{x}}{x+t} \frac{1}{x-b} d x=\operatorname{Re} \frac{\pi i}{\sqrt{b}+\sqrt{t} i}>0
$$

где $t>0, b \in \mathbb{C} \backslash[0, \infty)$ (см. задачу 28.20 из [4], значение $\sqrt{b}$ выбирается в верхней полуплоскости).

В случае $p \geqslant 2$ полюсы наипростейших дробей $r(z)$, ограниченных единицей по норме $L_{p}[0, \infty)$, по-видимому, могут сколь угодно близко подходить к нулю.

Отметим еще раз, что вытекающая из теорем 4-6 невозможность приближения полюсов наипростейших дробей $r(z)$ к рассматриваемым множествам $\gamma$ (углам и лучам) при условии $\|r\|_{L_{p}(\gamma)} \leqslant 1$ вызвана неограниченностью этих множеств на комплексной плоскости. В случае ограниченной гладкой незамкнутой кривой $\gamma$ без самопересечений наипростейшие дроби плотны в любом пространстве $L_{p}(\gamma)$ с $p \in$ 
$[1, \infty)$ (см. [6], [7]), поэтому для любой точки $a \notin \gamma$ существует такая наипростейшая дробь $r_{a}(z)$, что

$$
\left\|-\frac{1}{z-a}-r_{a}(z)\right\|_{L_{p}(\gamma)} \leqslant 1,
$$

т.е. наипростейшая дробь $1 /(z-a)+r_{a}(z)$ ограничена единицей по норме $L_{p}(\gamma)$, а один из ее полюсов - точка $a$ - может подходить к кривой $\gamma$ сколь угодно близко. В $\S 7$ работы [8] построены даже наипростейшие дроби $r_{n}(z)$, у каждой из которых все полюсы расположены в $\varepsilon_{n}$-окрестности отрезка $[-1,1](n=1,2, \ldots)$, и при этом $\varepsilon_{n} \rightarrow 0$ и $\left\|r_{n}\right\|_{L_{p}[-1,1]} \rightarrow 0$ при $n \rightarrow \infty, 1 \leqslant p<2$.

Автор благодарен рецензенту за полезные замечания.

\section{СПИСОК ЦИТИРОВАННОЙ ЛИТЕРАТУРЫ}

[1] В.И. Данченко, "Оценки расстояний от полюсов логарифмических производных до прямых и окружностей”, Матем. сб., 185:8 (1994), 63-80.

[2] П.А. Бородин, О.Н. Косухин, "О приближении наипростейшими дробями на действительной оси”, Вестн. Моск. ун-та. Сер. 1. Матем., Мех., 2005, № 1, 3-8.

[3] Дж. Гарнетт, Ограниченные аналитические функиии, Мир, М., 1984.

[4] М. А. Евграфов, Ю. В. Сидоров, М. В. Федорюк, М. И. Шабунин, К. А. Бежанов, Сборник задач по теории аналитических функиий, ред. М. А. Евграфов, Наука, М., 1969.

[5] Математическая энциклопедия, т. 1, М., 1977.

[6] В. И. Данченко, Д. Я. Данченко, "О равномерном приближении логарифмическими производными многочленов", Теория функций, ее приложения и смежные вопросы, Тезисы докл. школы-конференции, Изд-во Казан. ун-та, Казань, 1999, 74-79.

[7] В. И. Данченко, Д. Я. Данченко, “О приближении наипростейшими дробями”, Матем. заметки, 70:4 (2001), 553-559.

[8] В. И. Данченко, "Оценки производных наипростейших дробей и другие вопросы”, Матем. сб., 197:4 (2006), 33-52.

\section{П. А. Бородин}

Поступило

Московский государственный университет

26.12.2006

им. М. В. Ломоносова

E-mail: jborodina@inbox.ru 